\title{
AVALIAÇÃO DOS EFEITOS ANTITUMORAIS DE NOVOS CARDIOTÔNICOS ESTEROIDAIS SINTÉTICOS E SEUS EFEITOS NA NA,K-ATPASE
}

Oliveira, G.C. ${ }^{1 *}$, Ferreira, L.G.R. ${ }^{1}$, Neves, L.D.R. ${ }^{1}$, Grego, S.L.A. ${ }^{2}$, Villar, J.A.F.P. ${ }^{2}$, Santos, H.L. ${ }^{1}$, Barbosa, L.A. ${ }^{1}$, Cortes, V.F. ${ }^{1}$

${ }^{1}$ Lab. de Bioquímica Celular, Universidade Federal de São João Del-Rei, Divinópolis/MG

${ }^{2}$ Lab. de Síntese Orgânica, Universidade Federal de São João Del-Rei, Divinópolis/MG

*e-mail:gi_capanema@hotmail.com

\section{Resumo}

O efeito antitumoral de cardiotônicos esteroidais (CTS) tem sido observado em diversas linhagens celulares. CTS, como a digoxina e digitoxina, em doses terapêuticas possuem um efeito inibitório da Na,K-ATPase. Novos CTS sintetizados a partir da digoxina não possuem caracterização biológica na modulação da atividade da Na,K-ATPase nem seu potencial efeito antiproliferativo em células tumorais. $\mathrm{O}$ objetivo do estudo foi verificar o efeito citotóxico de novos compostos CTS sintéticos em células de carcinoma de colo uterino (HeLa) e cólon intestinal retal (RKO) e correlacionar o efeito antitumoral com a atividade da Na,K-ATPase. As células HeLa e RKO foram cultivadas e o ensaio de MTT foi realizado. As células HeLa foram tratadas com 6 novos CTS sintéticos nas concentrações de $150 \mathrm{nM}$ e $10 \mu \mathrm{M}$ por $24 \mathrm{~h}$. Como controle de inibição da Na,K-ATPase, foi utilizado a digoxina (150 nM). Após o tratamento, foi realizada preparação de membrana plasmática e a atividade da Na,K-ATPase. A análise estatística foi realizada no GraphPad Prism 5. Os novos CTS sintéticos DGB2, DGB3, DGB4, DGB5, DB6 e DGB7 apresentaram efeitos citotóxicos em células HeLa e RKO. Todos os compostos apresentaram IC50 na faixa de $50 \mu \mathrm{M}$, sendo o DGB5 o único que apresentou IC50 de 0,26 \pm $0,06 \mu \mathrm{M}$. O tratamento de $24 \mathrm{~h}$ com $150 \mathrm{nM}$ digoxina provocou $63 \%$ de inibição da Na,K-ATPase nas células HeLa. A inibição significativa da atividade da ATPase foi $96 \%$ a $10 \mu \mathrm{M}$ de DGB5, 50\% a $150 \mathrm{nM}$ de DGB5 e $46 \%$ a $10 \mu \mathrm{M}$ de DGB2. Nossos dados demonstram que o efeito citotóxico destes compostos não está relacionado com a inibição da atividade da $\mathrm{Na}, \mathrm{K}$-ATPase. O único 
composto que apresenta essa correlação é o DGB5, que apresentou o melhor IC50 para viabilidade celular e o melhor efeito de inibição da Na,K-ATPase.

Palavras-chave: Cardiotônicos esteroidais, Na,K-ATPase, Antitumorais Apoio financeiro: FAPEMIG, SBBq, UFSJ 